\title{
PERFIL DOS USUÁRIOS ATENDIDOS EM AMBULATÓRIO DE CUIDADO COM FERIDAS*
}

\author{
Regina Helena Squizatto ${ }^{1}$, Rosana Marcelino Braz ${ }^{2}$, Andressa de Oliveira Lopes ${ }^{3}$, Bruna Prini Rafaldini ${ }^{4}$, \\ Diego Bonil de Almeida ${ }^{5}$, Nadia Antonia Aparecida Poletti ${ }^{6}$
}

\begin{abstract}
RESUMO: O objetivo da pesquisa foi caracterizar o perfil dos pacientes atendidos em ambulatório de cuidado com feridas. Trata-se de estudo descritivo, retrospectivo, quantitativo, realizado em ambulatório de cuidado com feridas no interior do estado de São Paulo, com população de 347 prontuários, no ano de 2012 a 2014. Como resultado, houve predominância do sexo masculino (207/ 59,6\%), idade entre 19 e 59 anos (56\%) e casados (151/ 43,5\%). Observou-se que 203 (58,5\%) apresentavam alguma comorbidade e 103 (29,6\%) algum tipo de hábito, principalmente tabagismo e etilismo. Com relação às características da ferida, 225 (64,9\%) apresentavam apenas uma ferida e $90(26 \%)$ do tipo deiscência de sutura, $176(46,2 \%)$ das lesões localizavam-se no tronco e o tratamento mais utilizado foi com hidrogéis (153/21,3\%). Concluiu-se que o conhecimento do perfil da população permite ao enfermeiro realizar planejamento específico de assistência à saúde, evidenciando a importância deste profissional no cuidado às feridas.
\end{abstract}

DESCRITORES: Cuidados de enfermagem; Pacientes ambulatoriais; Cicatrização de feridas; Epidemiologia.

\section{PROFILE OF USERS ATTENDED AT A WOUND CARE OUTPATIENT CLINIC}

\begin{abstract}
The objective was to characterize the profile of the patients attended at a wound care outpatient clinic. A descriptive, retrospective and quantitative study was undertaken at a wound care outpatient clinic in the interior of the state of São Paulo, in a population of 347 patient histories, between 2012 and 2014. As a result, the following characteristics were predominant: male sex (207/59.6\%), age between 19 and 59 years $(56 \%)$ and married (151/43.5\%). It was observed that $203(58.5 \%)$ presented some comorbidity and $103(29.6 \%)$ some type of habit, mainly smoking and alcohol consumption. As regards the wound characteristics, 225 (64.9\%) presented only one wound and $90(26 \%)$ of the suture dehiscence type, $176(46.2 \%)$ of the lesions were located on the upper body and the most used treatment involved hydrogels (153/21.3\%). In conclusion, knowing the population profile allows the nurse to specifically plan health care, evidencing the importance of this professional in wound care.
\end{abstract}

DESCRIPTORS: Nursing care; Outpatients; Wound healing; Epidemiology.

\section{PERFIL DE LOS USUARIOS ATENDIDOS EN AMBULATORIO DE CUIDADO CON HERIDAS}

RESUMEN: El objetivo de la investigación fue caracterizar el perfil de los pacientes atendidos en ambulatorio de cuidado con heridas. Se trata de estudio descriptivo, retrospectivo, cuantitativo, desarrollado en ambulatorio de cuidado con heridas en el interior del estado de São Paulo, con población de 347 archivos, entre 2012 y 2014. Como resultado, predominaron el sexo masculino (207/ 59,6\%), edad entre 19 y 59 años (56\%) y casados (151/43,5\%). Se observó que 203 (58,5\%) presentaban alguna comorbilidad y 103 (29,6\%) algún tipo de hábito, principalmente tabaquismo y etilismo. Respecto a las características de la herida, 225 (64,9\%) presentaban solamente una herida y $90(26 \%)$ del tipo dehiscencia de sutura, $176(46,2 \%)$ de las lesiones se localizaban en el tronco y el tratamiento más utilizado fue conhidrogeles (153/ 21,3\%). Se concluyó que el conocimiento del perfil de la población permite al enfermero la planificación específica de la atención de salud, evidenciando la importancia de este profesional en el cuidado a las heridas. DESCRIPTORES: Atención de enfermería; Pacientes ambulatorios; Cicatrización de heridas; Epidemiología.

*Artigo extraído da dissertação intitulada: "Caracterização dos pacientes atendidos em um ambulatório de enfermagem de cuidados com feridas". Faculdade de Medicina de São José do Rio Preto, 2016.

${ }^{1}$ Enfermeira. Mestranda em Enfermagem. Faculdade de Medicina de São José do Rio Preto. Enfermeira da Unimed. São José do Rio Preto, SP, Brasil.

${ }^{2}$ Enfermeira. Pós-graduanda em Enfermagem em Dermatologia pela Faculdade de Medicina de São José do Rio Preto. Enfermeira da Unimed. São José do Rio Preto, SP, Brasil.

${ }^{3}$ Enfermeira, Mestranda em Enfermagem pela Faculdade de Medicina de São José do Rio Preto. Enfermeira na empresa Empório. São José do Rio Preto, SP, Brasil.

${ }^{4}$ Enfermeira. Mestranda em Enfermagem pela Faculdade de Medicina de São José do Rio Preto. Coordenadora Científica da Hollister do Brasil. São Paulo, SP, Brasil.

${ }^{5}$ Enfermeiro. Mestre em Enfermagem pela Faculdade de Medicina de São José do Rio Preto, Enfermeiro dermatológico da Unimed. São José do Rio Preto, SP, Brasil.

${ }^{6}$ Enfermeira. Doutora em Enfermagem. Docente da Faculdade de Medicina de São José do Rio Preto. São José do Rio Preto, SP, Brasil.

Autor Correspondente:

Recebido: $13 / 09 / 2016$

Regina Helena Squizatto

Finalizado: 06/02/2017

Faculdade de Medicina de São José do Rio Preto

R. Santina Figliagi Ceccato, 451 - 15035-180 - São José do Rio Preto, SP, Brasil

Email: squizatt@gmail.com 


\section{- INTRODUÇÃO}

A pele constitui órgão que reveste todo o corpo com a finalidade de proteção contra o meio externo, identificação, absorção, excreção, sensibilidade, dentre outras funções importantes. Denominamos de ferida a ocorrência de lesão aberta no tecido cutâneo, sendo também definida por solução de continuidade da pele $\mathrm{e}^{(1-2)}$.

Fatores classificados como extrínsecos ou intrínsecos estão relacionados com a ocorrência de feridas. Os fatores extrínsecos são representados principalmente pelos traumas externos. Já os fatores intrínsecos são predominantemente associados às comorbidades, como Diabetes Mellitus (DM), Hipertensão Arterial Sistêmica (HAS), Obesidade, Neoplasias, Vasculopatias, dentre outras ${ }^{(3-4)}$.

O aumento da expectativa de vida e o envelhecimento são fatores relacionados com a maior frequência de doenças crônicas e comorbidades na população. A presença de comorbidades predispõe ao desenvolvimento de feridas complexas, de difícil cicatrização e que necessitam de abordagem multiprofissional para o seu tratamento. Levando em consideração os inúmeros fatores que estão relacionados ao aparecimento de feridas, reforça-se a importância da atuação do enfermeiro especialista e do conhecimento da população alvo ${ }^{(2-4)}$.

As feridas complexas podem ser classificadas como agudas ou crônicas. As agudas são comumente representadas por lesões que cicatrizam dentro do tempo esperado e de acordo com os estágios do processo de cicatrização. São exemplos as traumáticas e as cirúrgicas. Já as feridas crônicas são aquelas de longa duração ou que apresentam reincidência, e, normalmente, estão associadas às comorbidades. São exemplos as lesões diabéticas, úlceras vasculogênicas, feridas neoplásicas, dentre outras ${ }^{(3,5)}$.

Os impactos provocados pelas feridas na população são diversos, tais como, o aumento dos custos com o tratamento, tanto para as instituições de saúde quanto para as famílias. As lesões complexas, principalmente as crônicas, demandam atendimento interdisciplinar especializado, uso de produtos tecnológicos e de longo período de tratamento. Além disso, a presença destas lesões muitas vezes causa afastamento das atividades laborais. Assim, a qualidade de vida também é prejudicada, já que a realização de atividades cotidianas torna-se mais penosa, e muitas vezes o indivíduo com feridas necessita da ajuda de outras pessoas para a realização destas atividades ${ }^{(5-7)}$.

O papel do enfermeiro especialista no cuidado de indivíduos com feridas inclui a avaliação geral, por meio da anamnese e exame físico; a avaliação específica da ferida; escolha do produto a ser utilizado; acompanhamento e avaliação do tratamento. Diversas características das lesões devem ser levadas em consideração na escolha do produto para o seu cuidado. Devem ser avaliados e registrados o tipo de tecido presente, as dimensões da lesão, o volume e característica do exsudato, presença de odor e análise da borda e pele perilesional ${ }^{(8-9)}$.

Atualmente, no mercado há inúmeros produtos e novas tecnologias disponíveis para o cuidado com feridas. A escolha do melhor tratamento leva em consideração os fatores de risco e comorbidades apresentados pelo indivíduo, assim como as características das lesões e suas condições sociodemográficas. Portanto, verifica-se a necessidade de atendimento multiprofissional especializado, incluindo o cuidado do enfermeiro especialista, além da necessidade de se conhecer todas as características do paciente, a família e o ambiente em que vivem ${ }^{(5,8-9)}$.

Diante da revisão de literatura apresentada, buscou-se com esta pesquisa, responder à seguinte questão norteadora: Qual o perfil da população atendida em um ambulatório especializado no cuidado com feridas?

Destaca-se que o conhecimento das características dos usuários atendidos no ambulatório de cuidados com feridas é essencial do ponto de vista da compreensão do seu perfil e das modificações necessárias para a organização do serviço e a melhora da qualidade da assistência de enfermagem. Neste contexto, o objetivo deste trabalho foi caracterizar o perfil dos pacientes atendidos em um ambulatório especializado no cuidado com feridas. 


\section{METODOLOGIA}

Estudo quantitativo, descritivo e retrospectivo, realizado em ambulatório de cuidado com feridas, localizado em um município do interior do estado de São Paulo. Neste ambulatório são atendidos pacientes com feridas agudas e crônicas, geralmente complexas, que necessitam de acompanhamento especializado.

O ambulatório está alocado no Ambulatório de Especialidades do Hospital de Base de São José do Rio Preto, estado de São Paulo, cuja abrangência representa todo o município e a região correspondente ao Departamento Regional de Saúde (DRS) XV, que consta de 102 municípios. A DRS XV faz parte da divisão administrativa da Secretaria de Estado da Saúde de São Paulo, com o objetivo de promover a regionalização das responsabilidades e articulação intersetorial. Além disso, também são atendidos usuários de outras regiões do país como Mato Grosso, Paraná e Minas Gerais, mostrando ser um serviço de referência no estado e no país. Neste ambulatório são realizados, em média, 600 atendimentos ao ano.

Foram incluídos no estudo todos os pacientes assistidos no serviço, no período de janeiro de 2012 a dezembro de 2014. Foram excluídos prontuários fora do período citado. Os dados foram obtidos por meio de pesquisa na base de dados do sistema eletrônico hospitalar, por intermédio do prontuário eletrônico do paciente. A coleta foi realizada no período de junho a agosto de 2015, por meio do instrumento de coleta de dados elaborado pelos pesquisadores, constando dados sociodemográficos e clínicos.

Para obtenção de informações sociodemográficas foram consideradas as seguintes variáveis: sexo, idade, estado civil, cidade de residência e escolaridade. Quanto às características clínicas, foram selecionadas as variáveis: comorbidades, hábitos (etilismo e tabagismo), tipos e número de feridas presentes, localização das lesões e terapias utilizadas.

Os resultados foram agrupados e organizados em Tabelas no programa Microsoft Excel 2007 e processados pelo programa Bioestat 5.3 por meio de estatística descritiva; sendo apresentados números absolutos e relativos das variáveis, seguidas de análise.

O estudo está vinculado ao projeto de pesquisa intitulado "Caracterização dos pacientes atendidos em um ambulatório de Enfermagem de cuidados com feridas", aprovado em 09 de fevereiro de 2015 pelo Comitê de Ética em Pesquisa com seres humano da Faculdade de Medicina de São José do Rio Preto (FAMERP); número de parecer 949.814.

Em virtude de dificuldades durante a coleta de dados, como a falta de informações em alguns prontuários, tais como diagnóstico médico, profissão do paciente e frequência de troca de curativos, estas informações coletadas não foram adicionadas nas planilhas e não aparecem nos resultados, com o objetivo de não comprometer a análise estatística. Este fato pode ser abordado como uma limitação do estudo.

\section{RESULTADOS}

No período de 2012 a 2014, foram atendidos 347 pacientes no referido ambulatório, constituindo a população do estudo, representando um total de 1.600 atendimentos. Conforme a Tabela 1, houve predominância de pacientes do sexo masculino. A idade variou entre 07 e 96 anos, apresentando média de 54 anos, com desvio padrão de 19 e coeficiente de variação de 35,4\%. Com relação ao estado civil houve predominância de casados (151/ 43,5\%).

Constatou-se prevalência de pacientes provenientes de 58 cidades que pertencem ao Departamento Regional de Saúde (DRS) XV. Levando-se em consideração a escolaridade, observou-se prevalência do ensino fundamental incompleto, representado por $143(41,2 \%)$. 
Tabela 1 - Distribuição das características sociodemográficas dos pacientes atendidos no ambulatório especialista de cuidado com feridas. São José do Rio Preto, SP, Brasil, 2012-2014

\begin{tabular}{|c|c|c|}
\hline Variáveis & Frequência & $\%$ \\
\hline \multicolumn{3}{|l|}{ Sexo } \\
\hline Masculino & 207 & 59,6 \\
\hline Feminino & 140 & 40,4 \\
\hline \multicolumn{3}{|l|}{ Idade } \\
\hline $0-11$ & 4 & 1,1 \\
\hline $12-18$ & 7 & 2 \\
\hline 19-38 & 72 & 20,8 \\
\hline $39-59$ & 122 & 35,2 \\
\hline 60 ou mais & 142 & 40,9 \\
\hline \multicolumn{3}{|l|}{ Estado civil } \\
\hline Casado & 151 & 43,5 \\
\hline Solteiro & 95 & 27,4 \\
\hline Divorciado & 29 & 8,4 \\
\hline Viúvo & 35 & 10 \\
\hline União estável & 35 & 10 \\
\hline Sem informação & 2 & 0,7 \\
\hline \multicolumn{3}{|l|}{ Procedência } \\
\hline São José do Rio Preto & 135 & 39 \\
\hline Cidades da região & 212 & 61 \\
\hline \multicolumn{3}{|l|}{ Escolaridade } \\
\hline Não alfabetizado & 36 & 10,3 \\
\hline Sem informação & 22 & 6,3 \\
\hline Fundamental incompleto & 143 & 41,2 \\
\hline Fundamental completo & 50 & 14,4 \\
\hline Médio incompleto & 18 & 5,2 \\
\hline Médio completo & 63 & 18,2 \\
\hline Superior incompleto & 4 & 1,2 \\
\hline Superior completo & 11 & 3,2 \\
\hline
\end{tabular}

Observou-se que elevado número dos pacientes possuía pelo menos uma comorbidade (203/ $58,5 \%)$, representado por mais da metade da população. A principal comorbidade foi a HAS (73/ 35,9\%) e a associação entre a HAS e o DM (51/25,1\%). As demais comorbidades foram representadas por Dislipidemias (DLP), seguidas de Insuficiência Vascular Periférica, Anemia, Insuficiência Renal Crônica (IRC) e a Osteomielite Crônica, observadas em 68 pacientes. Dentre os pacientes que apresentavam algum hábito, 50 (48,5\%) eram tabagistas, Tabela 2.

Tabela 2 - Distribuição dos pacientes atendidos no ambulatório especialista de cuidados com feridas de acordo com as comorbidades e hábitos presentes. São José do Rio Preto, SP, Brasil, 2012-2014

\begin{tabular}{lcc} 
Variáveis & Frequência & $\%$ \\
\hline Comorbidades & & \\
\hline Sim & 203 & 58,5 \\
\hline Não & 144 & 41,5 \\
\hline Tipo de comorbidade(1) & & \\
\hline DM* $^{*}$ & 25 & 12,3 \\
\hline
\end{tabular}




\begin{tabular}{lcc}
\hline HAS $^{* *}$ & 73 & 35,9 \\
\hline DM $^{*}+\mathrm{HAS}^{* *}$ & 51 & 25,1 \\
\hline Neoplasia & 57 & 28 \\
\hline Outras (2) & 76 & 37,4 \\
\hline Hábitos & & \\
\hline Sim & 103 & 29,6 \\
\hline Não & 244 & 70,4 \\
\hline Tipo de hábito(1) & & \\
\hline Tabagismo & 50 & 48,5 \\
\hline Etilismo & 30 & 29,1 \\
\hline Ex-tabagismo & 39 & 37,8 \\
\hline Ex-etilismo & 22 & 21,3 \\
\hline Drogas ilícitas & 4 & 3,8
\end{tabular}

(1) O mesmo paciente pode apresentar mais de uma comorbidade ou hábito.

(2) Infecção urinária de repetição, insuficiência renal crônica, asma, anemia, hepatite C, lúpus eritematoso sistêmico, osteomielite, AIDS, obesidade, insuficiência vascular periférica, tuberculose, Síndrome de Goldenhar, hipotireoidismo, insuficiência cardíaca congestiva, cirrose hepática, talassemia, doença de chagas, doença pulmonar obstrutiva crônica, dislipidemias.

*Diabetes Mellitus

**Hipertensão Arterial Sistêmica

Levando em consideração as características clínicas das feridas, verificamos a prevalência de pacientes com uma lesão (225/64,9\%). Quanto ao tipo de ferida encontrada, houve predominância de deiscência de sutura (90/26\%), seguida de lesão por pressão (85/24,5\%) e lesão traumática $(71 / 20,5 \%)$. Estes três tipos de feridas representaram $246(71 \%)$ do total de lesões estudadas. Com relação à localização das feridas, constatou-se que 334 (87,7\%) localizavam-se nos Membros Inferiores (MMII) e no tronco, apresentado na Tabela 3.

Tabela 3 - Distribuição dos pacientes atendidos no ambulatório especialista de cuidados com feridas, de acordo com o número e tipo de feridas, localização e produtos utilizados. São José do Rio Preto, SP, Brasil, 2012-2014 (continua)

\begin{tabular}{lcc} 
Variáveis & Frequência & $\%$ \\
\hline Número de feridas & & \\
\hline 1 & 225 & 64,9 \\
\hline 2 & 63 & 18,2 \\
\hline 3 ou mais & 59 & 16,9 \\
\hline Tipo de ferida & & \\
\hline Deiscência de sutura & 90 & 26 \\
\hline LPP* & 85 & 24,5 \\
\hline Traumática & 71 & 20,5 \\
\hline Tumoral & 18 & 5,2 \\
\hline Abscesso & 15 & 4,3 \\
\hline Úlcera venosa & 13 & 3,7 \\
\hline Outras & 55 & 15,8 \\
\hline Localização das lesões(1) & & \\
\hline MMII* & 158 & 41,5 \\
\hline MMSS*** & 14 & 3,7 \\
\hline Tronco & 176 & 46,2 \\
\hline Cabeça & 23 & 6 \\
\hline
\end{tabular}




\begin{tabular}{|c|c|c|}
\hline Pescoço & 10 & 2,6 \\
\hline \multicolumn{3}{|l|}{ Produtos utilizados nos curativos(2) } \\
\hline Hidrogéis & 153 & 21,3 \\
\hline Papaína & 138 & 19,2 \\
\hline Sulfadiazina de prata $1 \%+\quad$ nitrato de cério $0,4 \%$ & 99 & 14 \\
\hline Aloe vera gel & 81 & 11,3 \\
\hline $\mathrm{AGE}^{* * * *}$ & 64 & 8,9 \\
\hline Placa antimicrobiana & 59 & 8,2 \\
\hline Outros(3) & 123 & 17,1 \\
\hline
\end{tabular}

(1)Cada paciente pode apresentar mais de uma lesão em localizações diferentes.

(2)Cada paciente pode ter sido tratado com mais de um tipo de produto.

(3)Barbatimão, fator de crescimento, extrato glicólico de gengibre, creme barreira, soro fisiológico 0,9\%, hidratante de ureia a $10 \%$.

*Lesão por pressão

**Membros Inferiores

$* * *$ Membros Superiores

****Ácidos Graxos Essenciais

Ainda na Tabela 3 podemos observar que os principais produtos utilizados no tratamento das lesões foram: hidrogéis (153/ 21,3\%), incluindo hidrogel associado ao Polihexametileno Biguanida (PHMB) e hidrogel associado ao alginato de cálcio; papaína $(19,2 \%)$ e sulfadiazina de prata a $1 \%$ associado a nitrado de cério a $0,4 \%$ (99/ 14\%).

\section{- DISCUSSÃO}

De acordo com os resultados apresentados, predominaram no estudo pacientes idosos do gênero masculino, apresentando lesões crônicas. De acordo com a literatura, observa-se que o homem procura menos os serviços de saúde para promoção e prevenção de agravos. Isto pode ser observado em estudo realizado na região sul do país, em ambulatório de prevenção e tratamento de HAS, com prevalência de mulheres atendidas neste serviço ${ }^{(10)}$. Assim, a análise dos dados permite inferir que pessoas do sexo masculino procuram os serviços de saúde mediante a evidência de agravos à saúde.

No presente estudo, as feridas traumáticas representaram número importante com relação ao total (20,5\%), além disso, grande parte das feridas ocorreu nos membros inferiores (MMII) (56,2\%). Levandose em consideração a análise da relação entre a idade, o gênero e a ocorrência de feridas traumáticas na população, pode-se relacionar estudo realizado no estado do Piauí, com prevalência de acidentes com motocicleta em pacientes jovens, entre 18 e 29 anos e do sexo masculino. Além disso, grande parte destas lesões $(41,5 \%)$ ocorreu nos MMII, corroborando com os resultados deste trabalho ${ }^{(11)}$.

Estas características podem ser explicadas pela inexperiência e a incapacidade de se perceber o perigo pelos jovens ${ }^{(11)}$. Neste contexto, os profissionais de saúde têm grande importância na educação para prevenção de acidentes. Além disso, o conhecimento das causas das lesões permite realizar o planejamento de ações de prevenção.

Ainda evidenciando a importância da idade na relação com a origem da lesão, pode-se observar em trabalho realizado em Belo Horizonte que a minoria das lesões $(6,3 \%)$ era de origem traumática e a maioria (68,3\%) associada às doenças crônicas, sendo que a população mais frequente foi de idosos. Já no presente trabalho, o resultado foi diferente, um dos tipos mais prevalentes de lesão foi a traumática, sendo a população prevalente de jovens-adultos. Assim, a análise permite verificar que pacientes mais jovens possuem a tendência de apresentar feridas de origem traumática e pacientes idosos de feridas crônicas ${ }^{(2,11-12)}$.

Neste sentido, com o objetivo de modificar este quadro, é essencial o planejamento de estratégias de prevenção e promoção da saúde, com o objetivo de evitar o comprometimento da saúde decorrente das comorbidades, melhorando a qualidade de vida da população e reduzindo os gastos com saúde ${ }^{(5-7,13)}$. 
Continuando a análise das condições sociodemográficas da população, a maioria dos pacientes apresentou ensino fundamental incompleto, e residia em pequenas cidades da região, respectivamente $41,2 \%$ e $61,0 \%$. O baixo nível de escolaridade e o fato de residir em municípios pequenos podem representar recursos reduzidos e maiores dificuldades de acesso aos serviços de saúde, os quais influenciam na continuidade do regime terapêutico, dificultando também o autocuidado ${ }^{(5,14)}$.

Além disso, o entendimento das informações fornecidas pode ser prejudicado. Assim, as orientações durantes as consultas devem ser as mais claras possíveis, e o feedback de entendimento destas informações deve ser questionado, permitindo a continuidade do tratamento ${ }^{(5,14)}$.

Embora a ferida traumática represente, nesta pesquisa, 20,5\% do total da amostra, a mais frequente foi a deiscência de sutura $(26,0 \%)$, uma das complicações mais frequentes das feridas cirúrgicas. Alguns fatores podem influenciar na cicatrização de lesões cirúrgicas, tornando-a ferida complexa, com cicatrização por segunda intenção, necessitando de tratamento específico ${ }^{(15)}$.

A deiscência de ferida operatória compreende a ruptura parcial ou total do tecido epitelial e de planos profundos. A literatura apresenta vários fatores predisponentes desta condição, dentre elas, o Diabetes Mellitus (DM), a Hipertensão Arterial Sistêmica (HAS), o tabagismo e a infecção do sitio cirúrgico, que provocam alterações significativas no processo de cicatrização ${ }^{(15-17)}$. No presente trabalho, $73,3 \%$ dos pacientes que apresentaram alguma comorbidade tinham DM, HAS ou associação das duas doenças.

O excesso de glicose na corrente sanguínea provoca a redução da concentração de óxido nítrico sérico. Esta redução promove a disfunção endotelial, ocasionando um microambiente isquêmico periférico, que reduz a taxa de angiogênese. Além disso, promove a diminuição da resposta dos fatores de crescimento. Todas estas alterações culminam no retardamento do processo de cicatrização, favorecendo a ocorrência de deiscência de sutura nas feridas cirúrgicas ${ }^{(18)}$.

A elevação crônica da pressão arterial também provoca alterações microvasculares. Os vasos sanguíneos tendem a aumentar a espessura de sua parede, o que reduz seu lúmen. Assim, diminui o fluxo sanguíneo periférico, reduzindo o suprimento de oxigênio e nutrientes para a ferida. Além disso, a hipóxia local diminui a deposição de colágeno e inibe a ação fagocitária, provocando retardo na cicatrização ${ }^{(19)}$.

Quanto à presença de hábitos como o tabagismo e o etilismo, 86,3\% dos pacientes que tinham algum hábito declararam fazer uso do cigarro atualmente ou já terem sido tabagistas em algum momento da vida. A exposição crônica ao cigarro promove alterações vasculares importantes. A principal delas é a vasoconstrição periférica, que reduz o lúmen dos vasos sanguíneos e prejudica o aporte de oxigênio e nutrientes locais. Além disso, a nicotina, que representa o principal componente do cigarro, dificulta o transporte do oxigênio nas hemácias, agravando o quadro de hipóxia tecidual. A redução de oxigênio local, além de prejudicar a neoangiogênese e a multiplicação de fibroblastos nas feridas, propicia microambiente favorável para o crescimento de bactérias, aumentando o risco de infecção ${ }^{(20)}$.

Inúmeros aspectos estão relacionados com a ocorrência de feridas na população, como observado acima. Este fato aumenta a complexidade da avaliação, tratamento e acompanhamento destes pacientes. Mostra também a importância do conhecimento dos produtos disponíveis no mercado, assim como a indicação de cada um deles ${ }^{(9)}$.

Os principais objetivos de uma cobertura são controlar a carga microbiana, proteger de traumas, manter a umidade do meio favorecendo a multiplicação celular, e estimular a epitelização. Assim, todas as características das feridas devem ser avaliadas, tais como a extensão, profundidade, presença de túneis e fístulas, tipo de tecido, qualidade e quantidade da secreção, além da pele perilesional, permitindo assim a correta indicação dos produtos utilizados no tratamento ${ }^{(9)}$.

Atualmente, existe um quantitativo considerável de produtos no mercado com o intuito de favorecer o processo de cicatrização. Os principais produtos utilizados neste estudo foram: os hidrogéis, associados ao alginato de cálcio e ao Polihexametileno-biguanida (PHMB), a papaína e a sulfadiazina de prata a $1 \%$ mais nitrato de cério a $0,4 \%$, somando $54,5 \%$ dos produtos utilizados. Em quarto lugar ficou a utilização do gel de Aloe vera, seguido dos Ácidos graxos essenciais (AGE) e de placas antimicrobianas. Estudo realizado em ambulatório de cuidado com feridas do estado do Rio de 
Janeiro observou a utilização de hidrogel em 30\% dos pacientes, AGE em 23\% e colagenase em $16 \%{ }^{\left({ }^{(8)}\right.}$.

A escolha dos produtos a serem utilizados no tratamento de feridas leva em consideração, principalmente, as características das lesões. Grande parte dos produtos mantém o meio úmido, favorecendo a multiplicação e migração celular, além de promover o desbridamento autolítico. As placas como a hidrofibra com prata e de alginato de sódio e cálcio também apresentam a função de absorção da secreção em excesso, além de serem antimicrobianas em função da prata presente. A papaína e a colagenase, além de manter o meio úmido, possuem poder desbridante, favorecendo a retirada de tecidos desvitalizados por sua ação enzimática ${ }^{(8)}$.

$\mathrm{Na}$ escolha do produto mais adequado, além da avaliação da própria ferida, também deve ser levada em consideração a condição socioeconômica e de instrução dos pacientes. Um dos problemas enfrentados diz respeito ao alto custo de muitas terapias e a falta destes produtos na rede pública. Dessa forma, muitas vezes os pacientes necessitam comprar os produtos, o que compromete a renda familiar $^{(8)}$.

Em vista dos dados deste trabalho e os encontrados na literatura, pode-se destacar a importância de conhecer a população com a qual se cuida. As informações levam ao conhecimento das principais necessidades da população, permite otimizar o tratamento junto da utilização de produtos adequados e acessíveis. Admite também o planejamento de ações de promoção, prevenção e recuperação de saúde específica, de acordo com a necessidade deste grupo. Observa-se ainda, a importância da atuação do enfermeiro especialista, responsável pela análise de todos estes dados ${ }^{(8,21)}$.

Como limitação do estudo, pode-se destacar a coleta de dados através de prontuários, deixando o pesquisador dependente da inclusão de dados dos profissionais responsáveis pelo cuidado às feridas. Por este motivo, foi necessária a retirada de alguns dados na análise a fim de garantir a confiabilidade dos resultados.

\section{CONCLUSÃO}

O estudo permitiu traçar o perfil da população estudada. As principais características encontradas foram: pacientes do sexo masculino, idosos, casados, procedentes de cidades da região de São José do Rio Preto, com ensino fundamental incompleto e com deiscência de sutura (90/26\%), lesão por pressão $(85 / 24,5 \%)$ e lesão traumática $(71 / 20,5 \%)$.

Evidenciou-se que grande parte dos indivíduos que tinham feridas apresentavam comorbidades (58,5\%), sendo necessária a intervenção educacional. Este papel, fundamental do enfermeiro, permite evitar a ocorrência de complicações decorrentes das doenças pregressas. Ainda, foi possível observar o perfil de instrução da população, com o objetivo de intervir por meio de propostas educacionais acessíveis às características dos indivíduos.

Concluiu-se, portanto, que o conhecimento da população permite traçar algumas melhorias para a prática, como possibilitar o planejamento e execução de atividades específicas. Pode-se evidenciar a importância dos atendimentos em um ambulatório de especialidade no cuidado com feridas e a atuação do enfermeiro especialista. Este, responsável pela avaliação, conduta, execução e acompanhamento das lesões e dos pacientes, sempre levando em consideração todas as dimensões do indivíduo, físicas, emocionais, sociodemográficas e culturais.

\section{REFERÊNCIAS}

1. Rodrigues KLH, Leandro ALJS, dos Santos KFS, da Silva TCT, Guilherme FJA, dos Santos MSS. Identificação, tratamento e prevenção de feridas no neonato. Rev rede de cuidados em saúde. [Internet] 2015;9(2) [acesso em 04 jul 2016]. Disponível: http://publicacoes.unigranrio.edu.br/index.php/rcs/article/view/2599/1306.

2. Liedke DCF, Johann DA, Danski MTR. Consultório de enfermagem para tratamento de feridas em hospital de ensino. Cogitare Enferm. 2014;19(3):590-6.

3. Farina Junior JA, de AImeida CEF, Garcia FL, Lima RVKS, Marques RR, Cologna MHT. Tratamento multidisciplinar 
de feridas complexas. Proposta de criação de "Unidade de Feridas" no Hospital das Clínicas da FMRP-USP. Medicina (Ribeirão Preto). 2013;46(4):355-60.

4. Costa RKS, Torres GV, Salvetti MG, de Azevedo IC, da Costa MAT. Validade de instrumentos sobre cuidado de enfermagem à pessoa com lesão cutânea. Acta paul. enferm. 2014;27(5):447-57.

5. de Oliveira BGRB, Lima FFS, Araújo JO. Ambulatório de reparo de feridas - Perfil da clientela com feridas crônicas. Um estudo prospectivo. Online Braz J Nurs. [Internet] 2008;7(2) [acesso em 04 jul 2016] Disponível: http://www.objnursing.uff.br/index.php/nursing/article/view/j.1676-4285.2008.1508/369.

6. Albuquerque ER, Alves EF. Análise da produção bibliográfica sobre qualidade de vida de portadores de feridas crônicas. Revista Saúde e Pesquisa. 2011;4(2):147-52.

7. de Souza DMST, Borges FR, Juliano Y, Veiga DF, Ferreira LM. Qualidade de vida e autoestima de pacientes com úlcera crônica. Acta paul. enferm. 2013;26(3):283-8.

8. de Oliveira BGRB, Castro JBA, Granjeiro JM. Panorama epidemiológico e clínico de pacientes com feridas crônicas tratados em ambulatório. Rev enferm UERJ. 2013;21(n.esp 1):612-7.

9. Marques ADB, Guimarães MSO, da Cunha KAS, Cabral LAF, Queiroz AAFLN, de Oliveira LB. Critérios utilizados pelos enfermeiros na realização dos curativos. Rev. Pre. Infec e Saúde. 2015;1(1):31-9.

10. Cavalheiro AS, Fonseca MJ, Sbruzzi G, Goldmeier S. Perfil dos pacientes atendidos em um ambulatório de hipertensão arterial: há diferença entre os sexos?. Rev Gaúcha Enferm. 2014;35(1):110-5.

11. Soares LS, de Sousa DACM, Machado ALG, da Silva GRF. Caracterização das vítimas de traumas por acidente com motocicleta internadas em um hospital público. Rev enferm UERJ. 2015;23(1):115-21.

12. Maciel EAF, Carvalho DV, Borges EL, Matos SS, Guimarães GL. Prevalência de feridas em pacientes internados em hospital de grande porte. Rev enferm UFPI. 2014;3(3):66-72.

13. Rocha-Brischiliari SC, Agnolo CMD, Gravena AAF, Lopes TCR, Carvalho MDB, Pelloso SM. Doenças crônicas não transmissíveis e associação com fatores de risco. Rev Bras Cardiol. 2014;27(1):35-42.

14. de Oliveira FP, de Oliveira BGRB, Santana RF, Silva BP, Candido JSC. Classificações de intervenções e resultados de enfermagem em pacientes com feridas: mapeamento cruzado. Rev. Gaúcha Enferm. 2016;37(2):e55033.

15. Carvalho DV, Borges EL. Tratamento ambulatorial de pacientes com ferida cirúrgica abdominal e pélvica. Reme, Rev. Min. Enferm. 2011;15(1):25-33.

16. Silva BSM, Lua I, das Marces MC, de Souza e Silva D, Andrade LM, Oliveira DS. Cuidados de enfermagem na assistência domiciliar a um paciente com deiscência abdominal. Persp. Online: biol. \& saúde. 2014;15(4):1-6.

17. Tayeb KA. Managing infection: a holistic approach. J Wound Care. 2015;24(5 Suppl 2):20-30.

18. Lima MHM, Araujo EP. Diabetes mellitus e o processo de cicatrização cutânea. Cogitare Enferm. 2013;18(1):1702 .

19. Biondo-Simões MLP, Alcantara EM, Dallagnol JC, Yoshizumi KO, Torres LFB, Borsato KS. Cicatrização de feridas: estudo comparativo em ratos hipertensos não tratados e tratados com inibidor da enzima conversora de angiotensina. Rev. Col. Bras. Cir. 2006;33(2):74-8.

20. McDaniel JC, Browning KK. Smoking, chronic wound healing, and implications for evidence - Based practice. J Wound Ostomy Continence Nurs. 2014; 41(5):415-30.

21. Borges CL, da Silva MJ, Clares JWB, Nogueira JM, de Freitas MC. Características sociodemográficas e clínicas de idosos institucionalizados: contribuições para o cuidado de enfermagem. Rev enferm UERJ. 2015;23(3):381-7. 